\title{
Molten Salts as Stripping Media for Radioactive
}

\section{Superficial Decontamination}

\author{
Paulo Ernesto de Oliveira Lainetti \\ Nuclear and Energetic Research Institute (IPEN-CNEN/SP), Av. Prof. Lineu Prestes 2242, São Paulo/SP. CEP 05508-000, Brazil
}

Received: May 17, 2016 / Accepted: June 02, 2016 / Published: November 30, 2016.

\begin{abstract}
The main practical difficulty associated to the task of the dismantling and decommissioning of the IPEN's old nuclear fuel cycle facilities has been the big amount of radioactive waste generated in the dismantling operations. The waste is mainly in the form of contaminated carbon steel structures. In the IPEN, the presence of contamination in the equipments, structures and buildings, although restricted to low and medium activity levels, constituted an important concern due, on one hand, to the great volume of radioactive wastes generated during the operations. On the other hand, it should be outstanding that the capacity of radioactive wastes stockpiling in IPEN found been exhausted. In function of the large waste volume generated in the dismantling operations, the main concerns and focuses of research and technological development in the IPEN's Chemical and Environmental Center-CQMA have been the effluent and waste treatment subjects, besides the development of some special decontamination techniques, since most old nuclear fuel cycle facilities are installed in the CQMA's area. The reduction of the radioactive waste volume has a significant impact in the decommissioning costs and in the amount of material to be stored. The mentioned steel structures, during the operations and after ten or twelve years after the facilities shut down, have presented severe corrosion. In the past, to protect them, several layers of paint were applied. Traditional decontamination methods were tried, such as acid pickling, alkaline washing and ultrasonic baths. Nevertheless, these methods have failed to reach effective decontamination. In this paper, we described some aspects and problems in decommissioning of IPEN's nuclear fuel cycle facilities and it is presented an innovative method for radioactive superficial decontamination of steel structures using different molten salt compositions and temperatures as stripping media.
\end{abstract}

Key words: Molten, salts, stripping, radioactive, decontamination, decommissioning.

\section{Introduction}

One problem detected during the nuclear fuel cycle dismantling activities already performed in the IPEN was the difficulty of treatment of contaminated painted carbon steel structures. During the $70 \mathrm{~s}$ and $80 \mathrm{~s}$, IPEN built several facilities in pilot scale, destined to the technological domain of the several stages of the nuclear fuel cycle. In the nineties, radical changes in the Brazilian nuclear policy determined the interruption of the research activities and the plant-pilot's shut-down. Most of the pilot plants interrupted the activities in the beginning of nineties,

Corresponding author: Paulo Ernesto de Oliveira Lainetti, Ph.D., professor, metallurgist engineer, research fields: waste decomposition in molten salts, nuclear fuel cycle, thorium processing. due to the lack of resources for the continuity of the research. The appropriate facilities maintenance has been also harmed by the lack of resources, with evident signs of deterioration in structures and equipments. Those facilities also implicate in the need of constant surveillance, representing additional obligations, costs and problems.

In comparison with some developed countries, Brazil has a relatively modest and recent nuclear program. Due to the reduced dimensions of its nuclear market and to the lack of nuclear facilities shutdown projects in the near future, there still are no companies specialized in dismantling and decommissioning. Until 2000 year, the only decommissioning experience in Brazil was the closure of the Santo Amaro's Mill-USAM. This facility was installed in a 
residential area of S. Paulo (the largest city in South America) and with the property of Brazilian Nuclear Industry. During fifty years, it was dedicated to the processing thorium and rare earths from monazite sands from the southeast of Brazil. The operations were ended in 1992 and the decommissioning occurred between 1993 and 1999 [1].

In spite of several difficulties, such as lack of expertise and personnel dispersion in different activities, some facilities were actually dismantled at IPEN recently. Orthodox D\&D models/technologies could not be followed, because there is no prepared personnel for the function. Nevertheless, the operations were accomplished with a lot of radiological and environmental concerns, following strict procedures [2]. In the first phase of the D\&D activities, between 2003 and 2005, the main objectives were a preliminary rising of the nuclear facilities status, seeking the decommissioning. A preliminary report was prepared with the basic procedure to be adopted for the fuel cycle facilities dismantling at IPEN [3]. This rising allowed knowing each installation that should be decommissioned better, establishing a decommissioning strategy based on the institutional needs, besides trying to fill out the main gaps in terms of lack of appropriate technical knowledge to the decommissioning and to identify the main technical obstacles that would be faced in the facilities dismantling [4-7]. The dismantling operations were performed in four phases:

- In the 2000-2001, the Thorium Sulfate Dissolution and $\mathrm{UF}_{4}$ Production Pilot Plant-Aqueous Route in the Building 2 of CQMA were dismantled;

- Between 2002 and 2003, the ADU Dissolution (Impure Yellow Cake) and Uranyl Nitrate Purification Pilot Plants were dismantled;

- Between 2005 and 2006, the Uranium Hexafluoride Conversion Pilot Plant was dismantled [8];

- In 2007, the $\mathrm{UO}_{2}$ Pellets Pilot Plant [9] and prepared the plan for the decommissioning of the Pilot
Plants installed in the Building 2 of CQMA [10] was decommissioned.

During the activities described above, a large amount of radioactive waste was generated, mainly in the form of contaminated steel structures. This was the main practical difficulty associated to the task of the dismantling and decommissioning in IPEN. In accordance with the CNEN's (Brazilian National Nuclear Energy Commission) standards, this material was characterized as low level waste and the contamination was superficial. However, the total amount was in order of several tons, requiring a lot of drums and work for waste conditioning. The superficial contamination was consequence of the presence of the radionuclides: uranium of natural isotopic composition and/or thorium compounds. Several methods were attempted with the objective of superficial decontamination and the consequent reduction of the waste volume. However, the results were not satisfactory.

\section{Decontamination Methods Employed and Problems}

One problem detected during the accomplished dismantling activities was the difficulty of treatment of contaminated painted carbon steel structures. The operational conditions of the fuel cycle pilot plants were deleterious to structural materials, submitting them either to a chemically aggressive environment or to radioactive liquids and dust that formed deposits in the structure surfaces. The chemicals employed, like acids, caused accelerated corrosion of the carbon steel. To protect the structures and to increase their useful lives, it was employed painting as a protection agent. During the facility's operational life, several layers of paint were applied in an attempt to stop or reduce the corrosion rate, since the structures were submitted to aggressive environments. The presence of several layers of paint combined with corroded regions makes the decontamination process much more difficult, creating serious problems in terms of decontamination 
efficacy and workers exposition. In Fig. 1, it shows that the carbon steel structures employed in the pilot plants of IPEN, after their removal from the facilities, and the corrosion status of some structures after more than a decade since the interruption of the activities.

Several methods were attempted with the objective of superficial contamination removal and the consequent reduction of the contaminated waste volume, such as decontamination by pickling/rinsing treatments methods employing acid and alkali solutions (chemical method with nitric/citric acids or sodium hydroxide), combination of rinsing methods and ultrasonic device and abrasive removal (physical method). Different concentrations of such solutions were tested. Some tanks released in the dismantling operations were employed in the decontamination process to avoid the generation of new contaminated wastes. Besides the generation of large volumes of effluents that needed treatments by precipitation (for example, with sodium hydroxide added to $\mathrm{U}$ or Th acid solutions) and analysis before final disposal, the decontamination of some components was impossible. In some tests and measurements, it is possible to conclude that the radioactive contamination was concentrated mainly in the most corroded areas

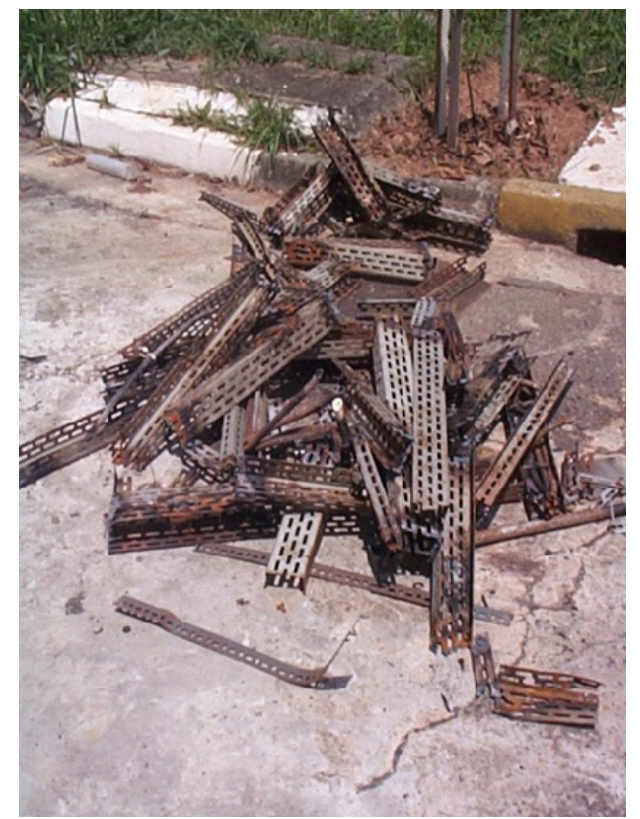

Fig. 1 Painted perforated carbon steel structures after the facilities dismantling. of the components. When paint was applied over these corroded areas, the acid or alkaline pickling was particularly impaired and the decontamination could not be accomplished satisfactorily.

Ultrasonic equipment available was also employed in an attempt to increase the efficiency of decontamination. However, the power of the equipment was not suitable for decontamination purposes. The equipment had some additional resources such as solution heating and bath agitation. The acid and alkaline solutions were again employed varying parameters such as concentration, temperature and time of immersion. In spite of the same good results obtained with different materials, as stainless steel, even for components with complex shapes, the results were frustrating to the painted carbon steel parts. The best results with stainless steel were obtained with nitric acid solutions, temperatures between $60-70{ }^{\circ} \mathrm{C}$ and ultrasonic support. In spite of some efficiency when applied to stainless steel components, those perforated, and several times painted carbon steel structures cannot be released by this method and all the waste had to be conditioned (cutting in suitable dimensions) and stored in special drums or steel boxes. The storage in drums is not suitable because it is necessary a lot of work to cut the structures in small pieces and the space is not completely fulfilled. The structures' storage in steel boxes is much more advantageous, since it is not necessary so much cutting work and the space is better occupied. Nevertheless, the boxes are expensive, there is a lot of free space and they still need large area for their storage.

To reduce the large volume of waste generated in the dismantling, some methods were tried as described above. For some materials, the available methods presented good results. For example, the case of stainless steel components, since more than 7,000 components with different shapes and sizes were effectively decontaminated during the operations. Good results were also obtained in the decontamination of aluminum components using a sodium hydroxide 
pickling solution. Nevertheless, the volume of waste in this case is not so significant. The radioactive waste constituted by painted and corroded carbon steel components deserves special attention, since the volume generated during the facilities dismantling was, at least, one order of magnitude bigger than the previously mentioned materials. Unfortunately, with the changes in the Brazilian R\&D Nuclear Program, the decontamination area in IPEN is not prepared to the treatment of the large waste volume generated during the D\&D activities. There are not tools and/or facilities to accomplish the needed operations.

As an attempt to determine the best available decontamination method, a literature review of methods for coating removal was accomplished. A very short summary of these methods is presented in this paper. In accordance with US EPA (United States Environment Protection Agency) [11], a cleaner technology is a source reduction or recycling method applied to eliminate or significantly reduce hazardous waste generation. Pollution prevention should emphasize source reduction technologies over recycling, but if source reduction technologies are not available, recycling is a good approach to reducing waste generation. The cleaner technology must reduce the quantity, toxicity or both of the waste produced.

\subsection{Coatings Removal Technologies}

Paint and other coatings are applied to surfaces to enhance corrosion resistance among other reasons. In our case, coating removal is required as part of the decontamination process, since the main objective of stripping the paint layers, applied to protect the carbon steel, is to permit the removal of the radioactive contamination from the surface from parts of the above mentioned structures. Solvent strippers have been widely used for paint and other coatings removal for many years. Solvent strippers can be applied at room temperature to remove a wide range of organic coatings without attacking metal substrates. Solvent strippers consist mainly of methylene chloride (60 to $65 \%$ of the formulation). Acid solvent strippers typically include phenol, formic acid and methanol monoethanolamine in the formulation in addition to methylene chloride. Use of solvent strippers generates organic vapors, sludge and wastewater containing solvents and metals. A wide range of environmental concerns about these release paths are leading industries to seek cleaner alternatives to coating removal. The main objective is to reduce significantly the release of VOCs (volatile organic compounds), particularly HAPs (hazardous air pollutants). Solvent waste disposal procedures and requirements increase waste management costs and establish cradle-to-grave responsibility. Besides this, methylene chloride can be considered as an occupational carcinogenic.

Then, different methods are available for effective, safe coating removal. These cleaner technologies use one or more of four types of physical mechanisms: Abrasive technologies - wear the coating off with scouring action; Impact technologies - rely on particle impact to crack the coating to remove it; Cryogenic technologies - use extreme cold to make the coating more friable and induce differential contraction to debond the coating; Thermal technologies - use heat input to oxidize, pyrolyze and/or vaporize the coating. Nine available cleaner coating removal technologies could be mentioned: plastic media blasting; wheat starch blasting; burn off coating removal; molten salt coating removal; sodium bicarbonate wet blasting; carbon dioxide pellet cryogenic blasting; high-pressure water blasting; medium-pressure water blasting; liquid nitrogen cryogenic blasting. Of course, different aspects should be considered to choose the most suitable technology among the mentioned above. Capital costs, complexity, required skill levels, number of steps involved, need of space are among the considerations to take into account before the final decision. Nevertheless, no one coating removal technology will be effective in all situations.

The choice of a coating removal process for radioactive wastes in the form of carbon steel pieces 
must take into account, among other factors, that it is not necessary a high quality of finishing, since the main objective is the release of the material as iron scrap. Different from other applications, where the main objective is to recover the component for reworking (appliances industry, for instance), the reduction of waste volume and the consequent need of expensive containers and space for storage are the driving forces. The presence of radionuclides as aerosols should be avoided since the contamination would spread out and the workers need to wear special clothes, respirators and eye protection equipment. Blasting also uses to generate high noise levels. Besides this, pieces with complex shapes need much work. In some cases, as internal parts (tubes), blasting is not effective.

\section{Molten Salt Stripping as a New Process for Radioactive Decontamination}

In spite of the use of molten salt has already been developed for some industrial coating removal process, it has not been found references in the literature about its use for radioactive superficial contamination removal. The molten salt stripping process relies on chemical oxidation of the coating by a molten salt bath. Then, the main objectives of this study are (considering the application of the method for radioactive waste treatment): development of specially formulated salt compositions that provide melting temperatures and cost as low as possible; optimization of the contact time between pieces and the salt bath to get the required contamination removal; development of a salt recycling method to reduce as much as possible the generation of secondary wastes.

As molten salt coating removal works by combusting the coating organics, the molten salt stripping process replaces solvent strippers. The organic content of the coating or paint (hydrocarbons) will be oxidized by reaction with air and the salt bath and will form only $\mathrm{CO}_{2}(\mathrm{~g})$ and $\mathrm{H}_{2} \mathrm{O}(\mathrm{v})$. In spite of the by-product used salt has a small volume containing mostly metal oxides and metal salts (formed by reaction of pigments with salt bath materials), the salt can be recycled. During molten salt stripping process, by-products of the reaction of the salt and the coating, as well as the radioactive contaminants present mainly in the corroded and oxidized areas of the metal surface, accumulate in the bath. Even when the bath is saturated with by-products, stripping will continue.

In the first set of experiments, 42 samples were cut from contaminated structures. Each sample was characterized by measurements of its activity in terms of the counts per second. As the activity is not constant along the piece, the maximum counts per second measured was adopted. Since the kind of structure (perforated steel in L form), material (carbon steel), dimensions and coating (painted) are always the same, the main process variables were selected: salt composition; salt temperature; residence time. Four different salt compositions were selected for the tests: pure sodium carbonate; pure sodium hydroxide; the eutectic mixture of sodium carbonate ( $41 \%$ in mass) and sodium hydroxide (59\% in mass); sodium hydroxide with addition of about $10 \%$ in mass of sodium nitrite (oxidizing salt). Two immersion or residence times were selected: 10 and 20 minutes. Temperatures selected were $450{ }^{\circ} \mathrm{C}$ and $650{ }^{\circ} \mathrm{C}$ for the different salt compositions and $900^{\circ} \mathrm{C}$ for pure sodium carbonate. The amount of salt in the reactor was approximately $3.5 \mathrm{~kg}$, corresponding to $40 \mathrm{~cm}$ height. Following immersion, the samples were removed from the salt bath and rinsed with water for salt removal and cooling. The rinsed items were measured again to determine their respective activities in terms of the maximum counts per second. After this step, the items were submitted to a pickling treatment with sulfuric acid $(98 \%)$ diluted in water ( $20 \%$ in volume). As showed in Fig. 2, the combustion of the paint creates a flame. The temperature of the bath of pure sodium carbonate was $900{ }^{\circ} \mathrm{C}$. The disadvantage of this compound is its relatively high melting temperature $852{ }^{\circ} \mathrm{C}$. The molten salt acts as a gas scrubber which retains the non-volatile reaction products (metal oxides 

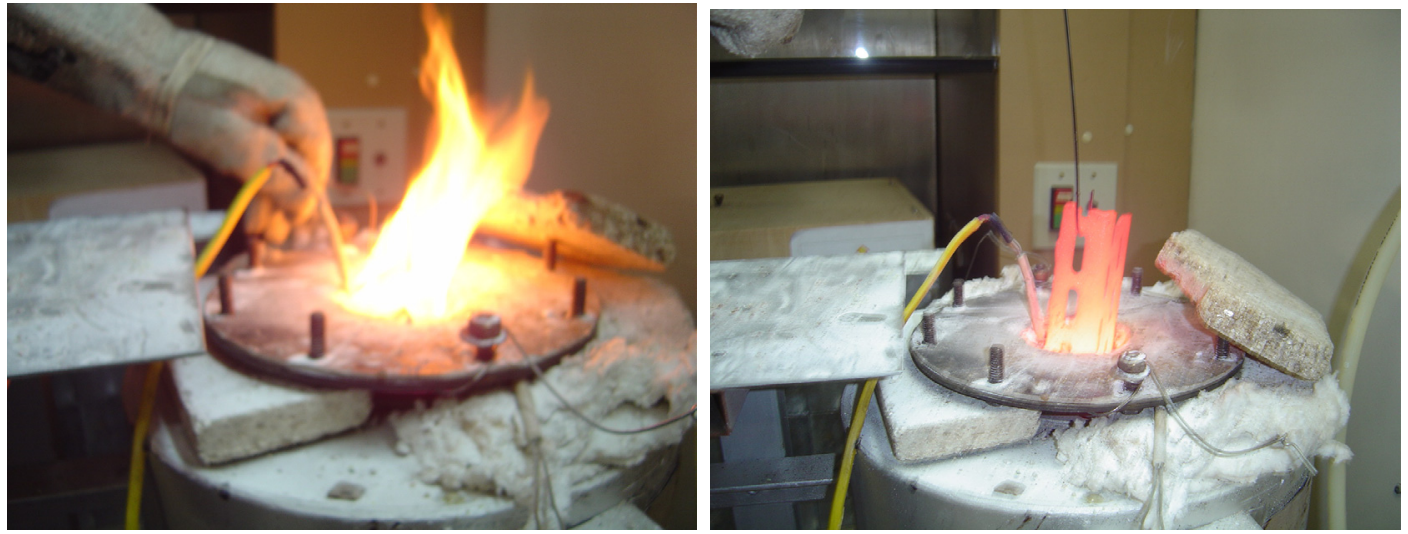

Fig. 2 Molten salt stripping—introduction (left) and removal (right) of a sample.

and ashes). As the main functions of the molten salts are a heat transfer medium and catalyst to oxidize the organics in the paint, and the costs of the process and some technical difficulties are associated to higher temperatures, it is important to remove the paint in temperatures as low as possible. Then, it is necessary to look for molten salt formulations that can provide low temperature and efficient paint removal.

\section{Conclusions}

The treatment of radioactive wastes in the form of painted structures, made of perforated carbon steel, and presenting superficial contamination has been investigated. The superficial radioactive contamination is located mainly in the corroded regions and mixed with rust. This contamination is difficult to removal due to the layers of paint applied on the part. The paint applied over the contamination impedes the action of the usual acid or alkaline pickling methods. The process selected for paint stripping was the immersion of pieces in molten salt mixtures in different temperatures and different residence times. Molten salt stripping uses simple and straightforward processing steps. The items to be stripped can be loaded into baskets or can be supported on hooks. The process allows rapid and complete painting removal with a minimum of handling. The method can be applied for parts with complex shapes. The superficial contamination of internal walls of tubes, which are not reached by blasting or abrasive methods, can be successfully treated by immersion in molten salts. Different molten salt compositions have been tested to determine the best salt formulation, since the temperature must be as low as possible. Sodium carbonates, sodium hydroxides, the eutectic mixture of sodium carbonate and sodium hydroxide and sodium hydroxide with addition of some oxidizing salts such as sodium nitrite were tested. The results obtained in terms of painting and contamination removal were excellent. Nevertheless, it is still possible to improve the salt mixture and optimize the salt temperature versus residence time. The exact mixture of salts can be tailored to achieve minimum operating temperatures and very short residence time.

\section{Acknowledgements}

On behalf of IPEN-CNEN/SP and myself, the author would like to express gratitude to the IAEA (International Atomic Energy Agency) for the financial support to the Research Project BRA 12800.

\section{References}

[1] Ferreira, P., Matta, L., and Mouço, C. 2006. "Decommissioning: Learned Lessons and Reference for the Future." In Proceedings of the International Conference on Lessons Learned from the Decommissioning of Nuclear Facilities and the Safe Termination of Nuclear Activities, 357-62.

[2] Lainetti, P. 2007. "Decommissioning of Nuclear Fuel Cycle Facilities in the IPEN-CNEN/SP." In Proceedings of the European Nuclear Conference, 110-4.

[3] Lainetti, P., Freitas, A., Ferrari, E., Ferreto, H., Ayoub, J. 
Seneda, J., and Bergamaschi, V. 2002. Plano de Ação para Desmontagem das Unidades do Ciclo do Combustivel. IPEN report, São Paulo (Action Plan for Dismantling of the Fuel Cycle Units).

[4] Lainetti, P., and Freitas, A. 2003. Desmontagem das Unidades de Dissolução e Purificação de Urânio do Ciclo do Combustivel, Bloco I CQMA. IPEN report, São Paulo.

[5] Lainetti, P. 2005. "Decommissioning of Nuclear Fuel Cycle Facilities-IPEN's Experience.” In Proceedings of the 7th Enan Meeting on Nuclear Applications, 216-22.

[6] Lainetti, P., Cotrim, M., Vasques, F., Ferreira, R., and Pires, M. A. 2007. "Reintegration of Areas of the IPEN's Dismantled Nuclear Fuel Cycle Pilot Plnats as New Laboratories of the CQMA." In Proceedings of the International Nuclear Atlantic Conference, R06_794: 1-6.

[7] Serviço de Radioproteção. 2003. Serviço de Radioproteção na Desmontagem e Descontaminação de Superficies da Instalação Nuclear de Purificação de Urânio e Processamento de Tório no IPEN/CNEN-SP.
IPEN report, São Paulo.

[8] Santos, I., Abrão, A., Carvalho, F. M. S., and Ayoub, J. M. S. 2009. "Decommissioning of an Uranium Hexafluoride Pilot Plant." In Proceedings of the International Nuclear Atlantic Conference, R06_892: 1-7.

[9] Cambises, P. B., Paiva, J., Almeida, C., Silva, T., and Rodrigues, D. 2007. "Radioprotection Planned Operation to Deactivate an Old Fabrication Plant of $\mathrm{UO}_{2}$ Pellets in IPEN-CNEN/SP to Perform Decommissioning." In Proceedings of the International Nuclear Atlantic Conference, E01_931: 1-8.

[10] Lainetti, P., Ferrari, E., Ayoub, J., Seneda, J., Yamasaki, A., Freitas, A., Santos, W., and Pires, M. A. 2007. Inventário dos Equipamentos das Usinas Piloto e dos Tambores de Rejeitos Armazenados no Bloco II CQMA-Estimativa de Custos da Desmontagem. IPEN report.

[11] US EPA. 1994. Guide to Cleaner Technologies-Organic Coating Removal. United States Environment Protection Agency, Washington DC, EPA/625/R-93/015. 